\author{
Lt Col MK GARG ${ }^{*}$, Lt Col KV BALIGA ${ }^{+}$
}

\begin{abstract}
MJAFI 2002; 58 : 53-59
KEY WORDS : Coronary artery disease (CAD); Coronary heart disease (CHD); Coalltion therapy; Hyperglycaemia; Oral hypoglycaemic agents (OHA)
\end{abstract}

D uring the past decade diabetes mellitus (DM), particularly type 2 diabetes has emerged as an important clinical and health problem. Several benefits to patients with diabetes are expected as a result of their treatment with improved patient education, availability of expertise, and sustained follow up and screening for the complications of diabetes. Several new groups of drugs have become available after initiation of historical UKPDS trial, thereby providing more flexibility in treatment and making the goal of glycemic control achievable. This article will outline current trends in the management of type 2 diabetes mellitus. However, managing diabetes is a difficult and time consuming task. It is common, serious, treatable and undertreated disease.

\section{How common is it?}

The epidemic is here in developing and newly industrialised nations. Between 1995 and 2025 there will be a $35 \%$ increase in the worldwide prevalence of diabetes. India will have second highest increase in prevalence of $59 \%$. The greatest increase will be seen in India, 195\% that is $19-57$ million [1]. At both points India will have the largest number of diabetics. More important is that from present position of harbouring one tenth of total world diabetics, India will harbour one fifth of all diabetics in the world by 2025 . More so, prevalence of diabetes is likely to increase with time in India, making the estimates even worse.

\section{How serious is it?}

Hyperglycaemia is the soil upon which CHD, CAD grow. Diabetic patients are at high risk of $\mathrm{CAD}$ progression, cardiovascular events and associated mortality. Females with diabetes had about four times and males $2 \frac{1}{2}$ times risk of developing CHD than nondiabetics [2]. Here is the setting of a CHD epidemic and disaster that will follow the epidemic of diabetes. The ratio of the mortality rates for diabetic versus nondiabetic were highest for those aged $25-44$ years with a value of 4.9 for men and 3.2 for women. The ratio declined with age but remained about $11 / 2$ times higher at the age group 65-74 years [3].

Microvascular complications add to morbidity related with diabetes duration and glycemic control. The 20 year prevalence of any type of retinopathy is about $80 \%$ in diabeties with poor glycemic control and $20 \%$ among those with acceptable diabetic control. The respective rates of proliferative retinopathy are $40 \%$ and $5 \%$. The risk of clinically significant macular edema is $10-15 \%$ after diabetes has been present for 15-20 years [4]. Those diabetics who had acceptable glycemic control about $80 \%$ remain free of microalbuminuria. However, in a patient with poor glycemic control, genetic predisposition determines the progression of diabetic nephropathy with time [5].

\section{Is it treatable?}

First and foremost aim is to prevent, delay, arrest or reverse diabetic complications and to improve quality and if possible, quantity of life. Kumamoto study [6], UKPDS Study [7] and DIGAMI study [8], have proved beyond doubt that various complications of diabetes can be prevented, arrested and delayed in type-2 diabetics. It is also possible to improve quality and quantity of life. Kumamoto study though had a small number of patients was just parallel in result with DCCT in IDDM patients. It has showed that intensified glycemic control by multiple insulin injection can delay the onset and the progression of diabetic microvascular complications. In UKPDS Study [7] patients assigned to intensive treatment had a $25 \%$ risk reduction in microvascular end points compared with conventional treatment. There was significant reduction in retinal photocoagulation and cataract extraction but had no effect on vitreous haemorrhage. There were 
significantly less number of patients with microalbuminuria, proteinuria and decreased biothesiometer perception in intensive group at the end of nine years. There was $16 \%$ reduction in risk for acute $\mathrm{MI}$ in UKPDS, though not reaching statistical significance. This may highlight the fact that probably glycemic control was not tight enough for macrovascular disease either, or glycemic threshold for progression is not known. In DIGAMI Study [8], patients with AMI with $B G>200 \mathrm{mg} / \mathrm{dl}$ were stratified in the control group and a group that received intravenous insulin infusion for first 24 hours and subsequently treated with subcutaneous insulin. Total mortality was $23 \%$ lower at 3 months and $29 \%$ at one year in insulin treated group.

\section{Stages in management}

Considering natural history of type-II diabetes, three stages can be defined in the management of diabetes with specific opportunity and tactics. These may be called public health care, primary physician care and specialist care. Public health care involves creating awareness about diabetes, conducting screening program for early diagnosis, and modifying life style of society to prevent or delay the onset of diabetes. Primary physician care till standard treatment protocols, fails to maintain acceptable glycemic control or intervention required for diabetes related complications. Specialised care to provide comprehensive care for complications and associated risk factors to decrease morbidity and mortality.

\section{Screening of Diabetes [9]}

To increase the cost effectiveness of testing undiagnosed diabetics, screening should be considered in high risk population eg. age $>45$ years, family history of diabetes (parent or sibling), obesity, hypertension, dyslipidemia or previous history of impaired glucose tolerance (IGT) or gestational diabetes mellitus (GDM). The screening test of choice is fasting plasma glucose (FPG). Fasting is defined as no consumption of food or beverages other than water for at least eight hours before testing. Here it should be noted that glucometer based capillary blood glucose is not recommended for screening. Screening of high-risk individual should be considered at 3 yearly intervals. The rationale for this interval is that there is little likelihood of an individual developing any complications of diabetes to a significant degree within 3 years of a negative screening test result. American Diabetes Association (ADA) has recommended three criteria for diagnosis of DM-FPG $>=126,2 \mathrm{hPG}>=200, \&$ $R P G>=200$ if symptoms are present . But it should be confirmed on a subsequent day by measurement of FPG or $2 \mathrm{hPG}$ again.

\section{What treatment modalities are available?}

There are four treatment modalities available, namely Medical Nutrition Therapy (MNT), excercise, oral drugs and insulin. Fifth modality though practised by almost every diabetic in India i.e. Ayurvedic, Homeopathic, advice of relatives and friends etc, hardly has thorough scientific basis. Moreover, diabetes is a progressive and life long disease hence any transitory experimental treatment should be discouraged.

Management of type 2 diabetes should follow staged and stepped approach beginning with life style modifications, oral hypoglycemic agents (OHA) should then be added singly or in combination [10]. Coalition therapy with OHA and insulin [11] and pure insulin therapy [12] should be given last to achieve appropriate glycemic control (Table-1).

TABLE

Metabolic control (ADA recommendation)

\begin{tabular}{lccc}
\hline Biochemical index & Good & Acceptable & Poor \\
\hline Fasting/preprandial plasma glucose & $80-120$ & $\leq 140$ & $>140$ \\
Postprandial plasma glucose & $80-160$ & $<180$ & $>180$ \\
Glycosylated Hb & $<8.5 \%$ & $8.5-9.5 \%$ & $>9.5 \%$ \\
\hline
\end{tabular}

\section{Life style modifications}

MNT and exercise are essential components of any treatment modality for diabetes mellitus. Without going into details of MNT, it is sufficient to stress that ethnicity of diet should be maintained rather than giving ready-made prescription. Diet should be modified with lowering caloric value, encouraging complex carbohydrate and fibres, and avoiding refined and free sugar. Daily brisk walk for half an hour should be encouraged in all after assessing underlying cardiac status.

\section{Single Oral Agents}

These consist of five groups of drugs - sulphonylurea, metformin, acarbose, thiazolidinediones and repaglinide.

Sulphonylureas : Sulphonylureas bind to an area of the beta cell plasma membrane associated with ATP dependent $\mathrm{K}^{+}$channels referred to as sulphonylurea receptor (SUR). Closure of $\mathrm{K}^{+}$channel depolarises the membrane and opens voltage dependent $\mathrm{Ca}^{+2}$ channel leading to exocytosis of insulin. The sulphonylureas differ in their potency per milligram, extent of hepatic metabolism, hypoglycemic activity, renal excretion, peak and duration of action, side effects and cost (Ta- 
ble-2). Each pharmaceutical company attempts to differentiate its sulphonylurea to increase market share but the differences are subtle and there is little evidence that they have major impact on success of therapy. Sulphonylurea therapy should be started with lowest recommended dose except in very symptomatic and hyperglycaemic patient. Glibenclamide, glipizide and gliclazide are better absorbed and more effective if given 30 minutes before meals. Common side effects include hypoglycaemia and weight gain.

TABLE 2

\begin{tabular}{lrlcl}
\hline Drug & Daily dose & Duration & Renal excretion & Cost (Rs) \\
\hline Glibenclamide & $2.5-20$ & $16-24$ & 50 & 0.50 \\
Glipizide & $2.5-20$ & $12-16$ & 85 & 0.50 \\
Gliclazide & $80-320$ & $10-20$ & 70 & $3.00(6.50)$ \\
Glimepiride & $1-8$ & 24 & 60 & 11.00 \\
\hline
\end{tabular}

Two newer sulphonylureas require special mention in view of tall claims made by the drug companies. Gliclazide molecule has azabicyclo-octyl ring attached which is said to impart beneficial effects on platelet function and vascular phenomenon related to atherosclerosis in patients with diabetes, however, better and clinically established effect can be obtained by half a tablet of aspirin with much lower cost. Glimepiride [13], new in Indian market, binds to a $65-\mathrm{kDa}$ protein on the beta cell rather than to the 140 $\mathrm{kDa}$ protein targeted by other sulphonylureas. There is less interaction of glimepiride than glibenclamide with cardiovascular ATP dependent $\mathrm{K}^{+}$channel, rendering it more pancreatic specific than other sulphonylureas. Glimepiride results in lower insulin and C-peptide levels with similar glucose control, supposedly as a result of greater insulin-mimetic effect in peripheral tissue. Gastro intestinal absorption is complete without interference from concurrent meals and drug accumulation is not seen with decreased renal function. However, importance of these differences in general clinical use needs to be determined.

Metformin (Rs.0.65 per $500 \mathrm{mg}$ tab): Metformin reduces appetite and glucose transport across intestinal mucosa. Its main effect is to reduce endogenous hepatic glucose output, which is contributed by increased hepatic insulin sensitivity and inhibition of gluconeogenesis. It also increases peripheral insulin mediated glucose uptake and decreases fatty acid oxidation [14]. There is also antiatherogenic effect, which appears to be independent of the changes in glycaemia; it includes improved lipid profile with decrease in triglyceride $10-20 \%$, total \& LDL cholesterol by 5 -
$10 \%$, and increase in HDL cholesterol, increased fibrinolytic activity (reduces PAI-1), decreased platelet aggregation, increased erythrocyte deformability, decreased arterial wall smooth muscle cell growth and decrease in insulin resistance [15]. Clinical evidence of this is $39 \%$ lower risk $(\mathrm{p}=0.01$ ) of myocardial infarction (MI) in metformin treated group in UKPDS [16]. It is preferred initial therapy in obese type-2 diabetics, however, it is also found equally effective in non-obese diabetics. Metformin is best started with $500 \mathrm{mg}$ twice or thrice daily with meal, gradually increased to 2-3 gm. Gastrointestinal side effects like nausea, metallic taste and increased bowel frequency are common. Metformin therapy should be avoided in patients with renal, hepatic and cardiac impairment due to increased risk of lactic acidosis. Almost all brands cost 65 paise per 500 -mg tablet.

Acarbose (Rs 5.50 per $50 \mathrm{mg}$ tab) : It competitively binds to the carbohydrate binding region of alpha-glucosidase enzymes i.e. sucrase, maltase, dextrinase, and glucomylase, and interferes with digestion of oligosaccharides. Undigested carbohydrate spills into large intestine where bacteria metabolise carbohydrate to short chain fatty acid leading to flatulence, diarrhoea and abdominal discomfort. An approximate mean decrease in FPG of $20 \mathrm{mg} / \mathrm{dl}$, PPPG of $50 \mathrm{mg} / \mathrm{dl}$, and HbAlc of $0.86 \%$ is to be expected with maximum dose of $100 \mathrm{mg}$ three times during meals. It is most effective with high carbohydrate diet. It should be titrated as per Table- 3 to avoid side effects. Acarbose also causes significant fall in blood ammonia, increase in gut peristalsis, leading to improvement in clinical signs of encephalopathy when administered to patients with type 2 diabetes and cirrhosis [15].

TABLE 3

Acarbose dosing schedule [10]

\begin{tabular}{lccc}
\hline Week & BF & Lunch & Dinner \\
\hline 1 & - & - & $1 / 2 \mathrm{tab}$ \\
2 & $1 / 2 \mathrm{tab}$ & - & $1 / 2 \mathrm{tab}$ \\
$3-4$ & $1 / 2 \mathrm{tab}$ & $1 / 2 \mathrm{tab}$ & $1 / 2 \mathrm{tab}$ \\
5 & $1 / 2 \mathrm{tab}$ & $1 / 2 \mathrm{tab}$ & $1 \mathrm{tab}$ \\
6 & $1 \mathrm{tab}$ & $1 / 2 \mathrm{tab}$ & $1 \mathrm{tab}$ \\
$7-8$ & $1 \mathrm{tab}$ & $1 \mathrm{tab}$ & $1 \mathrm{tab}$ \\
9 & $1 \mathrm{tab}$ & $1 \mathrm{tab}$ & $2 \mathrm{tab}$ \\
10 & $2 \mathrm{tab}$ & $1 \mathrm{tab}$ & $2 \mathrm{tab}$ \\
$11-12$ & $2 \mathrm{tab}$ & $2 \mathrm{tab}$ & $2 \mathrm{tab}$ \\
\hline
\end{tabular}

Thiazolidinedione (TZD) [17]: The TZD are a unique class of compounds that exert direct effect on the mechanism of insulin resistance and result in improved insulin action and reduced hyperinsulinemia. 
TZD binds to PPAR- $\gamma$ (peroxisome proliferator activator receptor) and regulates transcription of various molecules required for insulin action and lipid metabolism in adipocyte, skeletal muscle and liver. The biological potency of various TZDs is highly correlated with their binding to receptor. Rosiglitazone (RST) binds 10 fold more strongly than pioglitazone, which in turn binds 10 -fold more strongly than triglitazone, paralleling the clinical doses of $2-8 \mathrm{mg}, 30-50$ $\mathrm{mg}$, and $200-800 \mathrm{mg}$. TZDs also oppose the action of tumour necrosis factor- $\alpha$, a potential mechanism in insulin resistance state. Troglitazone has been withdrawn from world market due to its hepatotoxicity. Presently pioglitazone and rosiglitazone are being used world over. RST is likely to be introduced in Indian market shortly. Maximum response with RST monotherapy is seen at 8 weeks, with fall in FPG of about $75 \mathrm{mg} / \mathrm{dl}$ almost equivalent to sulphonylureas. It also lowers free fatty acid, triglycerides, and increases HDL and LDL cholesterol. However, LDL particles become larger, more buoyant, and less prone to oxidative modification. Its beneficial effect on the development of atherosclerosis remains to be determined. RST is not metabolised by the hepatic cytochrome, hence in clinical studies abnormal liver chemistry appears about one-tenth the rate of troglitazone [18].

Repaglinide : (Rs3.50 per $0.5 \mathrm{mg}$ tablet) Repaglinide a carbamoylmethyl benzoic acid derivative, is a non-sulphonylurea short acting insulin secretogogue. It stimulates insulin secretion by binding to ATP sensitive potassium channel on pancreatic beta cells, distinct from that of sulphonylurea. It is absorbed quickly (5-10 min), making it suitable for preprandial dosing. Maximum plasma levels are reached in 30-60 min, and action is terminated rapidly due to short half-life ( 1 hour), hence reduces the risk of postprandial hypoglycaemic events. It is mainly metabolised by liver (98\%) and excreted via bile $(90 \%)$, hence can be used effectively in patients with mild to moderate renal insufficiency. It is started in the dose of 0.5-2 mg preprandially. In view of short duration of action it can be given according to number of meals providing more flexibility, hence will be useful in diabetics with irregular food habits. Maximum clinical effect is evident within one week of repaglinide therapy [19].

\section{Combination Oral Therapy}

Initial successful treatment with single oral agent is likely to fall in 3-5 years, or desired glycemic control may not be achieved by single agent when initial FPG is $>200 \mathrm{mg} / \mathrm{dl}$, hence the obvious next step is to add a second agent. Above group of drugs have different mechanisms of action and therefore can be combined to obtain additive effect. Moderate doses of two agents may achieve equal glycemic effect with fewer side effects than full doses of either agent alone. Most widely used oral combination is sulphonylurea with metformin. There are fewer studies of sulphonylurea plus acarbose, metformin plus acarbose, and others [10]. However, lack of studies should not deter the physician to use combination of drugs with different mechanisms of action in a given patient to achieve glycemic control.

\section{Coalition Therapy (OHA and Insulin)}

During last two decades combination therapy with insulin and OHA has become popular. There are two aspects of coalition therapy [11]. One, additional insulin therapy, patient with unacceptable glycemic control with OHA can be prescribed additional night time insulin therapy. Another, adjunctive OHA therapy, patients uncontrolled with or requiring high doses of insulin monotherapy can be given OHA in the hope to achieve better glycemic control and decrease in the doses of insulin. Additional insulin therapy has more patient acceptance, improved glycemic control, rapid glycemic control, improved lipid profile, decreased insulin requirement, less weight gain and cost effectiveness [20-29].

\section{Indication for Coalition}

Sulfonylurea treatment undergoes secondary failure at the rate of 5-20\% yearly. Consequently, treatment fails in 5-20 years. Hence patients on maximum doses of OHA therapy when not able to maintain glycemic control to the acceptable level for 3-6 months should be started on coalition therapy.

\section{Coalition Regimes}

Sulfonylurea and insulin therapy : It decreases fasting serum glucose by $50-80 \mathrm{mg} / \mathrm{dl}$ and glycosylated haemoglobin by $1-2 \%$ [20-24]. Glycemic control improves in patients who have the worst glycemic control except when fasting blood glucose level is more than $300 \mathrm{mg} / \mathrm{dl}$ and in patients with the greatest degree of obesity.

Metformin and Insulin Therapy : A recently conducted study compared metformin plus insulin with sulfonylurea plus insulin in obese and non-obese patients with type-2 diabetes [28]. In the non-obese group, metformin treatment gave similar glycemic control, but with lower insulin doses and with lower weight gain. In obese patients, glycemic control was 
better and weight loss was more in metformin group.

\section{Insulin Regimens}

There has been no consensus about the form of insulin, and dose of insulin used. Various studies have definitely pointed towards beneficial effects of evening use of insulin. However, time and form of the insulin used varied in different studies.

Fixed Low Dose : Single night-time intermediate acing insulin e.g. lente insulin or NPH insulin, at the dose of $0.1-0.2 \mathrm{U} / \mathrm{kg}$ subcutaneously at $10.00 \mathrm{PM}$. It can be increased by $2-5$ units fortnightly to maintain fasting plasma glucose level [25]. If more than 30-36 units of insulin are necessary to obtain good glycemic control, consider stopping OHA treatment and continue on insulin monotherapy. Two studies have reported requirement of insulin about 20-30 units with this regime $[21,23]$

Maximum Tolerated Dose : Starting insulin in the dose of 10 units, increased fortnightly by 5-10 units till fasting blood glucose is controlled [22]. Insulin doses required with this regime ranges from $30-70$ units.

Monitoring of blood glucose : Monitoring of blood glucose should be done fortnightly, fasting and predinner. Blood glucose measurement at $3.00 \mathrm{AM}$ is indicated with symptoms suggestive of night time hypoglycaemia.

Failure of Coalition therapy : Failure of coalition therapy is indicated by uncontrolled predinner blood glucose level $(>180 \mathrm{mg} / \mathrm{dl})$ despite acceptable levels of fasting blood glucose.

\section{Insulin Therapy}

Insulin has a definite role in treatment of NIDDM as coalition therapy or monotherapy [12]. It can control hyperglycaemia in almost all cases when given in appropriate doses. There is a general trend in improvement of metabolic state with change towards normalization of blood glucose and lipid levels. It also increases sense of physical and psychological well being. Insulin therapy in NIDDM may be required regularly or intermittently (Table-4).

\section{Insulin Regimes}

Mixed Split Regime : On failure of coalition therapy a mixture of intermediate and short acting insulin can be given twice daily half an hour before breakfast and dinner. Usual starting dose is $0.5 \mathrm{unit} / \mathrm{kg}$, divided as two-third of total dose in the morning and one-third in the evening. Each dose consists of two-third of intermediate acting insulin and one-third short acting insulin. Adjustment of doses is done as per blood glu-
TABLE 4

Indication of insulin therapy

\begin{tabular}{ll}
\hline Regular therapy & Intermittent therapy \\
\hline 1. Non obese NIDDM < 40 years & 1. NIDDM with poor control \\
2. OHA failure & 2. Infection \\
\begin{tabular}{ll} 
(i) Primary - $30 \%$ & 3. Perioperatively \\
(ii) Secondary - $5-10 \%$ per year & 4. Pregnancy \\
3. Allergy and intolerance to OHA & 5. Acute medical emergencies \\
4. Renal and hepatic disease & 6. Acute surgical emergencies \\
& 7. Acute metabolic complications \\
\hline
\end{tabular}
\end{tabular}

cose monitoring performed premeal once a week. This regime is quite popular due to its simplicity and adequacy to achieve good glycemic control. It is ideally suited for patients with regular life style.

Multiple Subcutaneous Regime : This regime is superior to conventional regime as demonstrated by $\mathrm{Ku}$ mamoto study [6]. Those who are motivated educated and lead erratic life style, glycemic control can be achieved by multiple subcutaneous injection of short acting insulin before meals and at night time intermediate acting insulin.

\section{Control of Post Prandial Hyperglycemia}

Postprandial hyperglycaemia may be associated with a significantly increased risk of adverse cardiovascular events, even in the presence of normal FBG levels. Many options for reducing postprandial hyperglycaemia are available [30] :-

1. Reduction of carbohydrate intake

2. Ingestion of complex carbohydrate, mixed meal and fibres

3. Alpha glucosidase inhibitors

4. Metformin

5. Thiazolidinediones

6. Glucagon like peptide -1

7. Insulin secretogogues-Repaglinide

8. Preprandial insulin-insulin analogue (Insulin Lispro)

\section{Future prospects [31-33]}

Diabetes mellitus is a complex, and heterogeneous disorder with a variety of pathophysiological processes resulting in alteration in several metabolic pathways eg. glucose overproduction because of enhanced glycogenolysis and gluconeogenesis and glucose under utilisation due to diminished insulin production and insulin ineffectiveness. With the benefit of strict blood glucose control, there was a surge in search for more effective and safer hypoglycaemic agents. With better understanding of the metabolic derangements in diabetes mellitus in recent years, it became possible to 
develop agents that act at various stages of the altered hormone fuel homeostasis. Some of the agents which have been tried in human and animal experimental studies are as follows :

1. Inhibitors of Gluconeogenesis : Hydrazomopropionic acid Acyl CoA derivatives Pyruvate analogues

2. Inhibitors of glycogenolysis : Dichloacetate

3. Inhibitors of fatty acid oxidation :

4. Gut Lipase inhibitors :

Acipimox, Clomoxir Orlistat

5. Alpha glucosidase inhibitors : Miglitol, Voglibose

6. Increase incretin effect :

7. Short acting nonsulphonylurea insulin secretogogues :

8. Insulin sensitisers : GLP- 1 and related peptides BTS 67582, A -4166

SDZ PGU 693, NN 2344. NN622, Oxadiazolidinedione analog YM440

9. Minerals :

Vanadyl Sulphate.

Magnesium, Chromium

Picolinate

10.Prolongation of insulin action: Phosphotyrosine phosphatase inhibitors IRTK Activators.

11. Enhancement of GLLTT-4 translocation/expression

\section{How much is undertreated ?}

In a recently conducted cross sectional DiabcareAsia-India study, which included 26 specialised diabetic centres across the country, involving 2269 patients, central laboratory HbAlc data was categorised according to ADA guidelines. The proportion of patients with HbAlc $>1 \%$ above the upper limit of normal range (ULN) was $77 \%$, and $>2 \%$ above ULN was $50 \%$ for type-2 patients [34].

\section{References}

1. King H, Aubert RE, Herman WH. Global burden of diabetes, 1995-2025. Diabetes Care 1998;21:1414-31.

2. Folsom AR, Szklo S, Stevens P, Liao F, Smith R, Eckfeldt JH. A perspective study of coronary heart disease in relation to fasting insulin, glucose, and diabetes : The atherosclerosis risk in community (ARIC) study. Diabetes Care 1997; 20: 935-42.

3. Gu K, Cowie CC. Harris MI. Mortality in adults with and without diabetes in a national cohort of the US population, 1971-1993. Diabetes Care 1998;21:1138-45.

4. Ferris FL, Davis MD, Aiello LM. Treatment of diabetic retinopathy. N Engl J Med 1999;341:667-8.

5. Ritz ER, Orth SR. Nephropathy in patients with type-2 diabetes mellitus. N Engl J Med 1999;341:1127-33.

6. Ohkuba Y, Kishikawa H, Araki E, Miyata T, Isami S, Motoyosyi $S$ et al. Intensive insulin therapy prevents the progression of diabetic microvascular complications in Japanese patients with non-insulin dependent diabetes mellitus. A randomized prospective 6-year study. Diabetes Res Clin Pract 1995;28:103-17.

7. UK Prospective Diabetes Study Group. Intensive blood-glucose control with sulphonylureas or insulin compared with conventional treatment and risk of complications in patients with type-2 diabetes (UKPDS 33). Lancet 1998;352:837-53.
8. Malmberg K, Ryden L, Hamsten A, Herlitz J, Waldenstrom $A$, Wedel $\mathrm{H}$. Effects of insulin treatment on cause specific one year mortality and morbidity in diabetic patients with acute myocardial infarction. DIGAMI study group. Eur Heart J 1996;17:1337-44.

9. ADA Recommendation: Screening for type-2 diabetes. Diabetes Care 1999:22:S20-S23.

10. Hirsch IB, Riddle MC. Current therapies for diabetes. Endocrinol Metab Clin North America. 1997;26:51 1-678.

11. Saini JS, Garg MK. Insulin and OHA : Days of coalition therapy. API Medicine Update 1999;9(II)287-99.

12. Garg MK, Saini JS. Insulin therapy in non insulin dependent diabetes mellitus. Golden Jubilee National Medical Update 1998;39-51.

13. Bloomgarden ZT. New and traditional treatment of glycaemia in NIDDM. Diabetic Care 1996;19:295-9.

14. Bailey CJ. Tumer RC. Metformin. N Engl J Med 1996; 334 : 574-9.

15. Bloomgarden ZT. Type-2 diabetics : its prevalence, causes and ureatment. Diabetes Care 1998;21:860-5.

16. UK Prospective Diabetes Study Group. Effect of intensive blood-glucose control with metformin on complications in overweight patients with type-2 diabetes (UKPDS 34). Lancet 1998;352:854-65.

17. Saitiel AR, Olefsky JM. Thiazolidinediones in the treatment of insulin resistance and type-2 diabetes. Diabetes 1996; 45 : 1661-9.

18. Bloomgarden ZT. Treatment of type-2 diabetes. Diabetes Care 1999:22:170-4.

19. Gomis R. Repaglinide as monotherapy in type-2 diabetes. Exp Clin Endocrinol Diabetes 1999:107(suppl 4):S133-S135.

20. Pugh JA, Wagner ML. Sawyer J. Ramirez G, Tuley M, Friedberg $\mathrm{SJ}$. Is combination sulfonylurea and insulin therapy useful in NIDDM patients? A meta analysis. Diabetes Care 1992:15:953-9.

21. Ykj-Jarvinen H, Kauppila M, Kujansuu E, Lahti J, Marjanen T. Niskanen $L$ et al. Comparison of insulin regimens in patients with non-insulin dependent diabetes mellitus. $\mathbf{N}$ Engl J Med 1992;327:1426-33.

22. Riddle MC, Scheider J (The Glimeperide Combination Group). Beginning insulin treatment of obese patients with evening 70/30 insulin plus glimeperide versus insulin alone. Diabetes Care 1998;21:1052-7.

23. Romano G, Patti L, Inneli F, Di-Marino L, Annuzzi G, Lavicoli $\mathrm{M}$, Coronel GA et al. Insulin and sulfonylurea therapy in NIDDM patients : Are the effects on lipoprotein metabolism different even with similar blood glucose control. Diabetes 1997:46:1601-6.

24. Johnson JL, Wolf SL, Kabadi UM. Efficacy of insulin and sulfonylurea combination therapy in type II diabetes : a meta analysis of randomised placebo controlled trials. Archh Intern Med 1996;156:259-64.

25. Edelman SV, White D, Henry RR. Intensive insulin therapy or patients with type-II diabetes. International Diabetes Monitor 1995;7:1-9.

26. Taskenen MR, Sane T, Helve E. Karone SL, Nikkila EA, Yki-Jarvinen H. Bedtime insulin for suppression of overnight free fatty acid. blood glucose, and glucose production in NIDDM. Diabetes 1989;38:580-8. 
27. Lebovitz HE. Alpha-glucosidase inhibitors. Endocr Metab Clin North Am 1997;26:539-52.

28. Robinson AC, Burki J, Robinson S. Johnston DG, Elkells RS. The effoct of metformin on glycemic control on serum lipids in insulin-treated NIDDM patients with suboptimal metabolic control. Diabetes Care 1998:21:701-5.

29. Giugliano D, Quatraro A. Consoli G, Minei A, Cerriello A, De-Rosa $\mathrm{N}$ et al. Metformin for obese, insulin treated diabetic patients : improvement in glycemic control and reduction of metabolic risk factors. Eur J Clin Pharmacol 1993;44:10712.

30. Ladgraf $\mathbf{R}$. Approaches to the management of postprandial hyperglycemia. Exp Clin Endocrinol Diabetes 1999; 107 (suppl14) : S128-S132.

31. Bloomgarden ZT. Type-2 diabetes. Diabetes Care 1997; 20 : 1791.5.

32. Bressler R, Johnson D. New phamacological approaches to therapy of NIDDM. Diabetes Care 1992:15:792-802.

33. Puri D. Mohapatra TK. New oral hypoglycemic agents : Merits and Demerits. Asian J Diabetology 2000:2:44-8.

34. A survey-study on diabetes management and diabetes complication status in Asian countries (Unpublished Report). 1998:38-40. 